\title{
Aspects of Pattern-matching in Data-Oriented Parsing
}

\author{
Guy De Pauw \\ CNTS \\ University of Antwerp
}

\begin{abstract}
Data-Oriented Parsing (DOP) ranks among the best parsing schemes, pairing state-of-the art parsing accuracy to the psycholinguistic insight that larger clunnks of syntactic structures are relevant grammatical and probabilistic units. Parsing with the Dop-model, however, seems to involve a lot of CPU cycles and a considerable amount of double work, brought on by the concept of multiple derivations, which is necessary for probabilistic processing, but which is not convincingly related to a proper linguistic backbone. It is however possible to reinterpret the jop-model as a pattern-matching model, which tries to maximize the size of the substructures that construct the parse, rather than the probability of the parse. By emphasizing this memory-based aspect of the DOP-model, it is possible to do away with multiple derivations, opening up possibilities for efficient Viterbistyle optimizations, while still retaining acceptable parsing accuracy through enhanced context-sensitivity.
\end{abstract}

\section{Introduction}

The machine learning paradigm of MemoryBased Learning, based on the assumption that new problems are solved by direct reference to stored experiences of previously solved problems, has been successfully applied to a number of linguistic phenomena, such as part-of-speech tagging, NP-chunking and stress acquisition (consult Daelemans (1999) for an overvicw). To solve these particular problems, linguistic information needed to trigger the correct disambiguation, is encoded in a linear feature value representation and presented to a memory based learner, such as TiMBL (Daelemans ct al., 1999).

Yet, many of the intricacies of the domain of syntax do not translate well to a linear representation, so that established MBL-methods are necessarily limited to low-level syntactic analysis, like the aforementioned NP-chunking task.
Data Oriented Parsing (Bod, 1999), a statcof-the art natural language parsing system, translates very well to a Memory Based Learning context. This paper describes a reinterpretation of the DOP-model, in which the pattern-matching aspects of the model are exploited, so that parses are analyzed by trying to match a new analysis to the largest possible substructures recorded in momory.

A short introduction to Data Oriented Parsing will be presented in Section 2, followed by an explanation of the term pattern-matching in the context of this paper. Section 4 describes the experimental setup and the corpus. The parsing phase that precedes the disambiguation phase will be outlined in Section 5 and a description of the 3 disambiguating models, PCFG, PMPG and the combined system PCFG+PMPG can be found in Sections 6,7 and 8 .

\section{Data Oriented Parsing}

Data Oriented Parsing, originally conccived by Remko Scha (Scha, 1990), has been successfully applied to syntactic natural language parsing by Rens Bod (1995), (1999). The aim of Datia Oriented Parsing (henceforth DOP) is to develop a performance model of natural language, that models language use rather than some type of competence. It adapts the psycholinguistic insight that language users analyze sentences using previously registered constructions and that not only rewrite rules, but complete substructures of any given depth can be linguistically relevant units for parsing.

\subsection{Architecture}

The core of a DOP-system is its TREEBANK: an annotated corpus is used to induce all substructures of arbitrary depth, together with their respective probabilities, which is a expressed by 

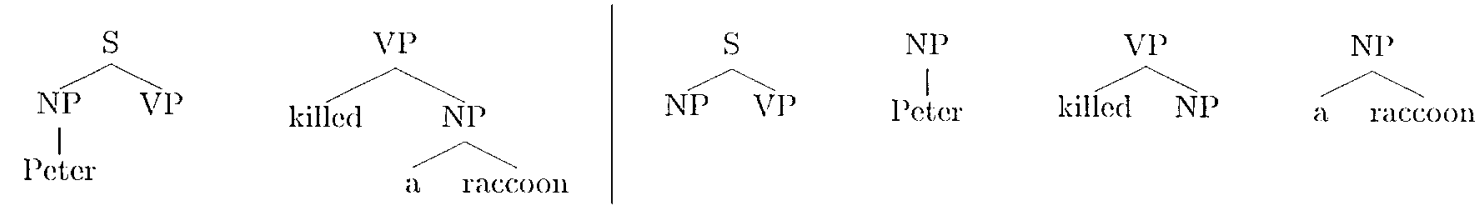

Figure 1: Multiple Derivations

its fiequency in the TREEIBANK relative to the number of substructures with the same rootnode.

Figure 1 shows the combination operation that is needed to form the correct parse tree for the sentence Peter killed a raccoon. Given a trecbank of substructures, the system tries to match the leftmost open node of a substructure that is consistent with the parse tree, with the top-node of another substructure, consistent with the parse tree.

Usually, different; combinations of substructures are possible, as is indicated in ligure 1: in the example at the left-hand side the tree-structure can be built by combining an sstructure with a specified NP and a fully specified vP-structure. 'I'he right example shows another possible combination, where a parse tree is built by combining the minimal substructures. Note that these are consistent with ordinary rewritc-rules, such as $\mathrm{S} \rightarrow \mathrm{NP}$ V1.

One particular parse tree maly thus consist of several different derivations. To find the probability of a derivation, we multiply the probatbilities of the substructures that were used to form the derivation. To find the probability of a parse, we must in principle sum the probabilities of all its derivations.

It is computationally lardly tractable to consider all derivations for cach parse. Since VI'TERBI optimization only succecds in findling the most probable derivation as opposed to the most probable parse, the MONTE CARLO algorithm is introduced as a proper approximation that randomly generates a large number of derivations. The most probable parse is considered to be the parse that is most often observed in this derivation forest.

\subsection{Experimental Results of nOP}

The basic Dop-model, Dopl, was tested on a manually edited version of the ATIS-corpus (Marcus, Santorini, and Marcinkiewic\%, 1993). 'The system was trained on 603 sentences (part- of-speech tag sequences) and evaluated on a test set of 75 sentences. Parse accuracy was used as an evaluation metric, expressing the percentage of sentences in the test set for which the parse proposed by the system is completely identical to the one in the original corpus. Jifferent experiments were conducted in which maximum substructure sire was varied. With DoP 1limited to a substructure-size of 1 (equivalent to a $P C F G$, parse accuracy is $47 \%$. In the optimal DOP-morkel, in which substructure-sizo is not limited, a parse accuracy of $85 \%$ is obtiained.

\subsection{Short Assessment of DOP}

Dor'1 in its optimal form achieves a very high parse accuarcy. The computational costs of the system, however, are equally ligh. Bod (1995) reported an average parse time of 3.5 hours per sentence. Even though current parse time is reported to be more reasonable, the optimal DOP algorithm in which no constrains are nade on the size of substructures, may not yet bo tractiable for life-size corpora.

In a context-free grammar framework (consistent with DOP limited to a substructure-sizo of 1), there is only one way a parse tree can be formed (for example, the right hand side of Figure 1), meaning that there is only one clerivation for a given parse tree. This allows efliciont VITERBI style optimization.

To encode context-sensitivity in the system, DOP is forced to introduce multiple derivations, so that repeatedly the same parse tree needs to be genorated, bringing about a lot of computattional overhear.

Even though the use of larger syntactic contexts is highly relevant, from a psycholinguistic point-of-view, there is no explicit preference being made for larger substructures in the DOI model. While the Mon'te CARLO optimization scheme maximizes the probability of the derivations and secms to prefer derivations made up of larger substructures, it may be interesting to 


\begin{tabular}{c|cc|c||cc}
\hline Disambiguator & Parse Accuracy $(/ 562)$ & $\%$ & F & Parse Accuracy on parsable sentences $(/ 456)$ & $\%$ \\
\hline PCFG & 373 & $\mathbf{6 6 . 4}$ & 83.0 & 373 & 81.8 \\
PMPG & 327 & 58.2 & 75.1 & 327 & 71.7 \\
PCFG+PMPG & 402 & $\mathbf{7 1 . 5}$ & 85.2 & 402 & 88.2
\end{tabular}

Table 1: Experimental Rosults

(a) Correct Analysis

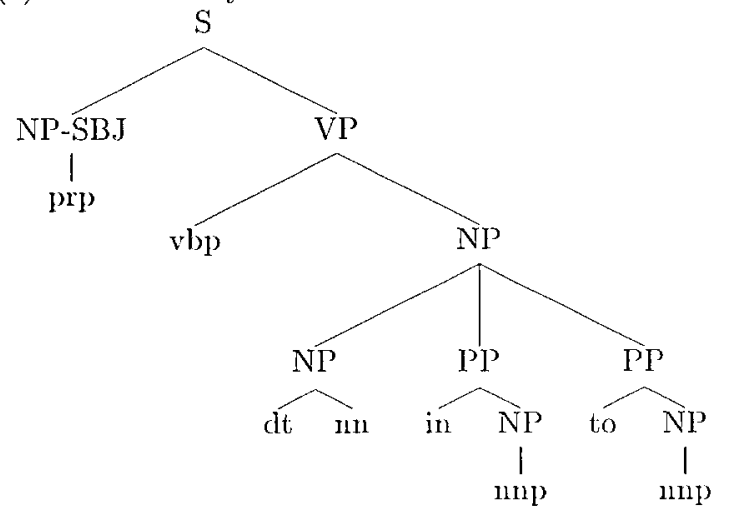

(b) PCFG-Analysis

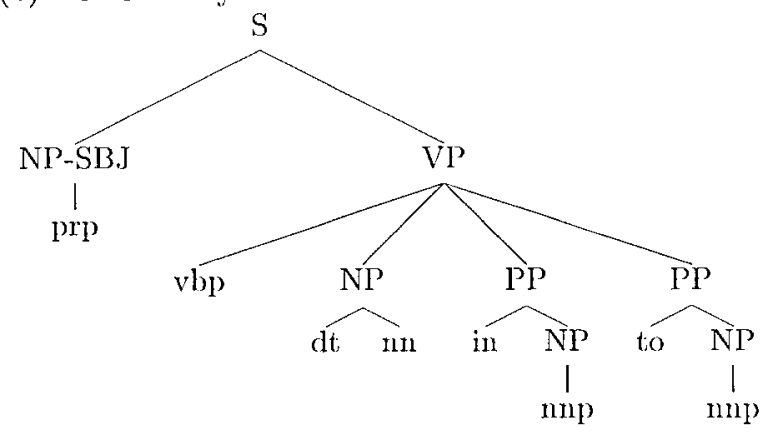

Figure 2: PCFG Error Analysis

sec if we can make this assumption explicit.

\section{Pattern-matching}

When we look at natural language parsing from a memory-based point of view, one might say that a sentence is analyzed by looking up the most similar structure for the different analyses of that sentence in memory. The parsing system described in this paper tries to mimic this behavior by interpreting the DOP-model as a memory-based model, in which analyses are being matched with syntactic patterns recorded in memory. Similarity between the proposed analysis and the patterns in memory is computed according to:

- the number of patterns needed to construct a tree (to be minimized)

- the size of the patterns that are used to construct a tree (to be maximized)

The nearest neighbor for a given analysis can be defined as the derivation that shares the largest amount of common nodes.

\section{The experimental Setup}

10-fold cross-validation was used to appropriately evaluate the algorithms, as the dataset (see Section 4.1) is rather small. Like DOP1 the system is trained and tested on part-of-speech tag scquences. In a first phase, a simple bottomup chart parser, trained on the training partitions, was used to generate parse forests for the part-of-speech tag sequences of the test partition. Next, the parse forests were sent to the 3 algorithms (henceforth the disambiguators) to order these parse forests, the first parse of the ordered parse forest being the one proposed by the disambiguator.

In this paper, 3 disambiguators are described:

- PCFG: simple Probabilistic Context-Free Grammar

- PMPG: the DOP approximation, PatternMatching Probabilistic Grammar

- PCFG+PMPG: a combined system, intcgrating PCFG and PMPG

The evaluation metric used is parse accuracy, but also the typical parser evaluation metric $F$ measure (precision/recall) is given as a means of reference to other systems.

\subsection{The Corpus}

The experiments were conducted on an edited version of the ATIS-II-corpus (Marcus, Santorini, and Marcinkicwicz, 1993), which consists of 578 sentences. Quite a lot of errors and inconsistencies were found, but not corrected, since we want our (probabilistic) system to be 
al)le to deal with this kind of noise. Semantically oriented flags like - T'MP and -DIr, most often used in conjunction with l'P, have been removed, since there is no way of retrieving this kind of semantic information fiom the part-ofspeech tags of the $\Lambda$ TIS-corpus. Syntactic flags like -SI3.J, on the other hand, lave been maintained. Internal relations (denoted by numeric flags) were removed and for practical reasons, sentence-length was limited to 15 words max. The edited corpus retained 562 sentences.

\section{Parsing}

As a first phase, a bottom-up (hart, parser parsed the tost set. This proved to be quite problematic, since overall, 106 out of 562 sentences $(19 \%)$ could not be parsed, clue to the sprarseness of the grammar, meaning that the appropriate rewsite rule needed to construct the correct parse tree for a sentence in the test set, wasn't featured in the induced grannmar. $\mathrm{NI}^{3}$ amotation seemed to be the main callse for: tu1parsalbility. $\Lambda \mathrm{n}$ NP like restriction code $A P / 57$ is represented by the rewrite rule:

$\mathrm{NP} \rightarrow$ NN NN sym sym sym CI CD

Highly specific and flat, structures like these are scarce ind are ustually not induced from the training set when needed to parse the test sot.

On-going rescarch tries to implement, grammatical smoothing as a solution to this problem, but one miglat, also consider generating parse forests with an independent grammar, induced from the entire corpus (training set-f testset) or a different corpus. In both cases, however, we would need to apply probabilistic smoothing to be able to assign probabilities to unknown structures/rules. Neither grammatical, nor probabilistic smoothing was implemented in the context of the experiments, described in this paper.

The sparseness of the grammar proves to be a serious bottleneck for parse accuracy, limiting our disambiguators to a maximum parse accuracy of $81 \%$.

\section{PCFG-experiments}

$\Lambda$ PCFG constructs parse trees by using simple rewrite-rules. The probability of a parse tree can be computed by multiplying the probabilities of the rewrite-rules that were used to construct the parse. Note that, a PCFG is identional to DOP 1 when wo limit the maximum substructures size to 1, only allowing derivations of the type found at the right-hand side of Figure 1.

\subsection{Experimental Results}

The first line of Table 1 shows the results for the PCFG-experiments: $66.4 \%$ parse accuracy is an adequate result for this baseline model. Wo also look at parse accuracy for parsable sentences (an estinnate of the parse accuracy we might get if we had a nore suited parse forest generator) and we notice that we are able to achieve a $81.8 \%$ parse accuracy. This is already quite high, but on examining the parsed data, serious and fundamental limitations to the PCFG-model (an be observed

\subsection{Error Analysis}

Figure 2, displays the most common type of mistake made by l'CrG's. The correct parse tree conld represent an analysis for the sentence:

$$
\text { I want a flight from Brussels to Toronto. }
$$

This example shows that a PCVG has a tendency to prefer flatter structures over embedded structures. This is a trivial effect of the mathematical formula used to compute the probability of a jarse-tree: embedded structure require more rewrite rules, adding more factors to the mn1tiplication, which will alnost inevitably result in a lower probability.

It is an mufortunate property of PCGG's that the number of nodes in the parse tree is inversely proportionate to its probability. One mighte be inclined to normalize a parse tree's probability relative to the number of nodes in the tree, but, a more linguistically somed alternative is at hand: the enhancement of context sensitivity through the use of larger syntactic context within parse trees can make our disambiguator more robust.

\section{PMPG-experiments}

The Patticrn-Matching Probabilistic Grammar is a memory-based interpretation of a nopmodel, in which a sentence is analyzed by matching the largest possible chunks of syntactic structure on the sentence. To compile parse trees into patterns, all substructures in the training set are encoded by assigning them specific: indexes, $\mathrm{NP}(\mathrm{Q}) 345$ e.g. denoting a fully specified NI-structure. This approach was inspired by Goodman (1996), in which Goodman 
unsuccessfully uses a system of indexed parse trees to transform DOP into an equivalent PCFG. The system of indexing (which is detailed in De Pauw (2000)) used in the experiments described in this paper, is however specifically geared towards encoding contextual information in parse trees.

Given an indexed training set, indexes can then be matched on a test set parse tree in a bottom-up fashion. In the following example, boxed nodes indicate nodes that have been retrieved from memory.

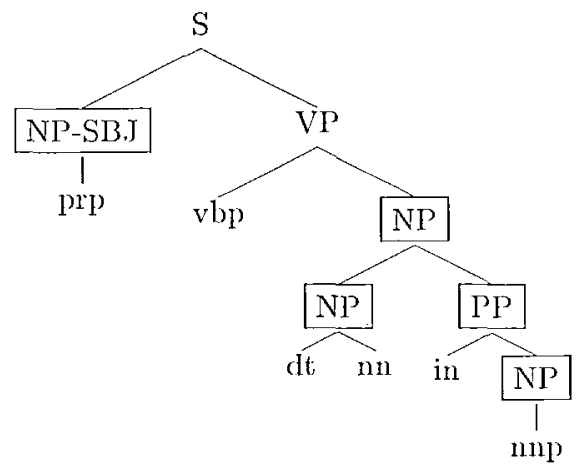

In this example we can see that an NP, consisting of a fully specified cmbedded $\mathrm{NP}$ and PP, has been completely retricved from memory, meaning that the NP in its entirety can be observed in the training set. However, no VP was found that consists of a VBP and that particular NP. Disambiguating with PMPG consequently involves pruning all nodes retrieved from memory:

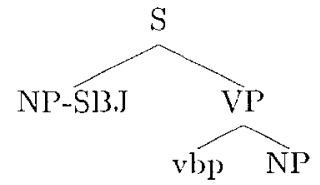

Finally, the probability for this pruned parse tree is computed in a PCFG-type manner, not adding the retrieved nodes to the product:

$$
\mathrm{P}(\text { parse })=\mathrm{P}(\mathrm{s} \rightarrow \mathrm{NP}-\mathrm{SB} . \mathrm{JP}) . \mathrm{P}\left(\mathrm{VI}^{\mathrm{P}} \rightarrow \mathrm{vbl}_{\mathrm{P}} \mathrm{NI}^{2}\right)
$$

\subsection{Experimental Results}

The results for the PMPG-experiments can be found on the second line of Table 1. On some partitions, PMPG performed insignificantly better than PCFG, but Table 1 shows that the results for the context sensitive scheme are much worse. $58.2 \%$ overall parse accuracy and $71.7 \%$ parse accuracy on parsable sentences indicatics that PMPG is not a valid approximation of DOP's context-sensitivity.

\subsection{Error Analysis}

The dramatic drop in parsing accuracy calls for an error analysis of the parsed data. Figure 3 is a prototypical mistake PMPG has made. Tho correct analysis could represent a parse tree for a sentence like:

What flights can I get from Brussels to Toronto.

The PMPG analysis would never have been considered a likely candidate by a common PCFG. This particular sentence in fact was offortlessly disambiguated by the PCFG . Yet the fact that large chunks of tree-structure are retrieved from memory, make it the preferred parse for the PMPG. We notice for instance that a large part of the sentence can be matched on an SBAR structure, which has no relevance whatsocver.

Clearly, PMPG overestimates substructure size as a feature for disambiguation. It's interesting however to sec that it is a working implementation of context sensitivity, cagerly matching patterns from memory. At the same time, it has lost track of common-sense PCFG tactics. It is in the combination of the two that one may find a decent disambiguator and accurate implementation of context-sensitivity.

\section{A Combined System (PMPG + PCFG)}

Table 1 showed that $81.8 \%$ of the time, a PCFG finds the correct parse (for parsable sentences), meaning that the correct parse is at the first place in the ordered parse forest. $\mathbf{9 9 \%}$ of the time, the correct parse can be found among the 10 most probable parses in the ordered parse forest. This opens up a myriad of possibilities for optimization. One might for instance use a best-first stratcgy to gencrate only the 10 best parses, significantly reducing parse and disambiguation time. An optimized disambiguator might therefore include a preparatory phase in which a common-sense PCFG retains the most probable parses, so that a more sophisticated follow-up scheme need not bother with senseless analyses.

In our experiments, we combined the common-sense logic of a PCFG and used its output as the PMPG's input. This is a wellestablished technique usually referrod to as system combination (see van Halteren, Zavrel, and Daelemans (1998) for an application of this 
technique to part-of-speech tagging):

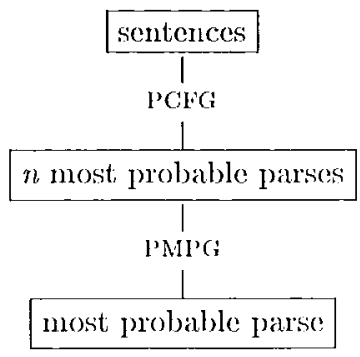

We are also presented with the possibility to assign a weight to each algorithm's decision. The probability of a parse can the be described with the following formula:

$$
P(\text { parse })=\frac{\prod_{i} P(\text { rewritc-rule })_{i}}{(\# \text { non-indexed nocles })^{n}}
$$

The weight of cach algorithm's decision, as well as the number of most probable parses that are extrapolated for the pattern-matching algorithm, are parameters to be optimized. Future work will include evaluation on a validation set to retrieve the optimal values for these parannetcrs.

\subsection{Results}

The third line in Table 1 shows that the combined system performs better than either one, with a parse accuracy of $71.5 \%$ and close to $90 \%$ parse accuracy on parsable sentences, which we can consider an approximation of results reported for DOP1. Error analysis shows that the combined system is indeed able to overcome difficulties of both algorithms. The example in Figure 2 as woll as the example in Figure 3 were disambiguated correctly using the combined system

\section{Future Research}

Even though the PMPG shows a lot of promise in its parse accuracy, the following extensions noed to be researched:

- Optimizing PMPG+PCFG for computational efficiency: the graph in Section 8 shows a possible optimized parsing system, in which a pre-processing PCFG generates the $n$ most likely candidates to be extraj)olated for the actual disambiguator. Full parse forests were gencrated for the experiments described in this paper, so that the efficiency gain of such a system cannot be properly estimated.

- PMPG +PCFG as an approximation needs to be compared to actual DOP, by having DOP parse the data used in this experiment, and by having PMPG + PCFG parse the data used in the experiments described in Bod (1999).

- 'The bottleneck of the sparse grammar problem prevents us from fully exploiting the disambiguating power of the patternmatching algorithm. The GRAEL-system (GRammar Adaptation, Evolution and Learning) that is currently being developed, tries to address the problem of grammatical sparscness by using evolutionary techniques to generate, optimize and complement grammars.

\section{Conclusions}

Even though DOr1 exhibits ontstanding parsing behavior, the efficiency of the model is rather problematic. The introduction of multiple derivations canses a considerable anount of computational overhead. Neither is it clear how the concept of multiple derivations translates to a psycholinguistic context: there is no proof that language users consider different instantiations of the sanne parse, when deciding on the correct analysis for a given sentence.

$\Lambda$ pattern-matching scheme was presented that tried to disambignate parse forests by trying to maximize the size of the substructures that can be retrieved from memory. This staightforward memory-based interpretation yiclds sub-standard parsing accuracy. But the combination of common-sense probabilities and enhanced context-sensitivity provides a workable parse forest disambiguator, indicating that language users might exert a complex combination of memory-based recollection techniques and stored statistical data to analyzo utterances.

\section{References}

Bod, R. 1995. Enriching linguistics with statistics: Performance models of natural language. Dissertation, Il, ,C, Universiteit van Ansterdam.

Bod, Rent. 1999. Beyond Grammar-An EaperienceBased Theory of Language. Cinnbridge, Hugland: Cambridge University Press.

Daelemans, W., J. Zavrel, K. Van eler Sloot, and A. Van den Bosch. 1999. TiMlBL: Tilburg Memory 
(a) Correct Analysis

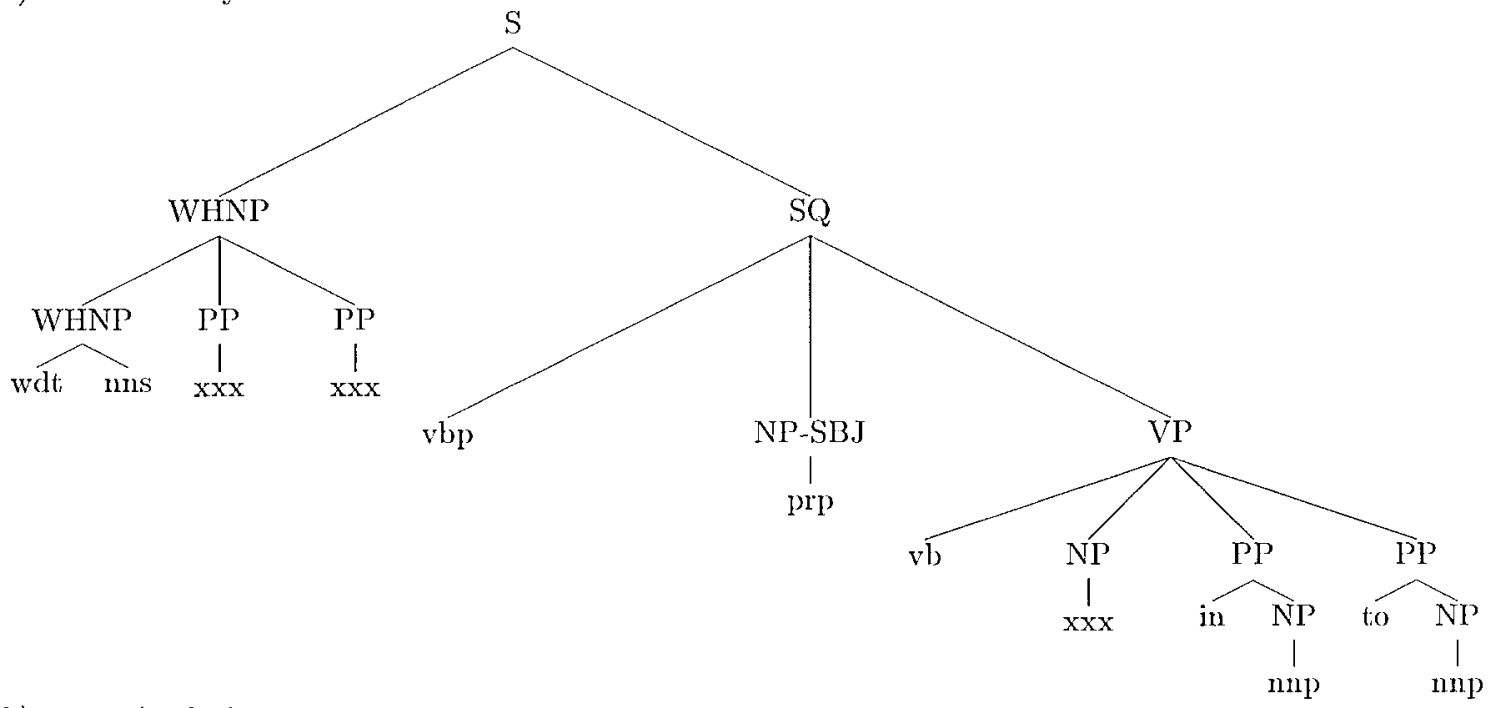

(b) PMPG Analysis

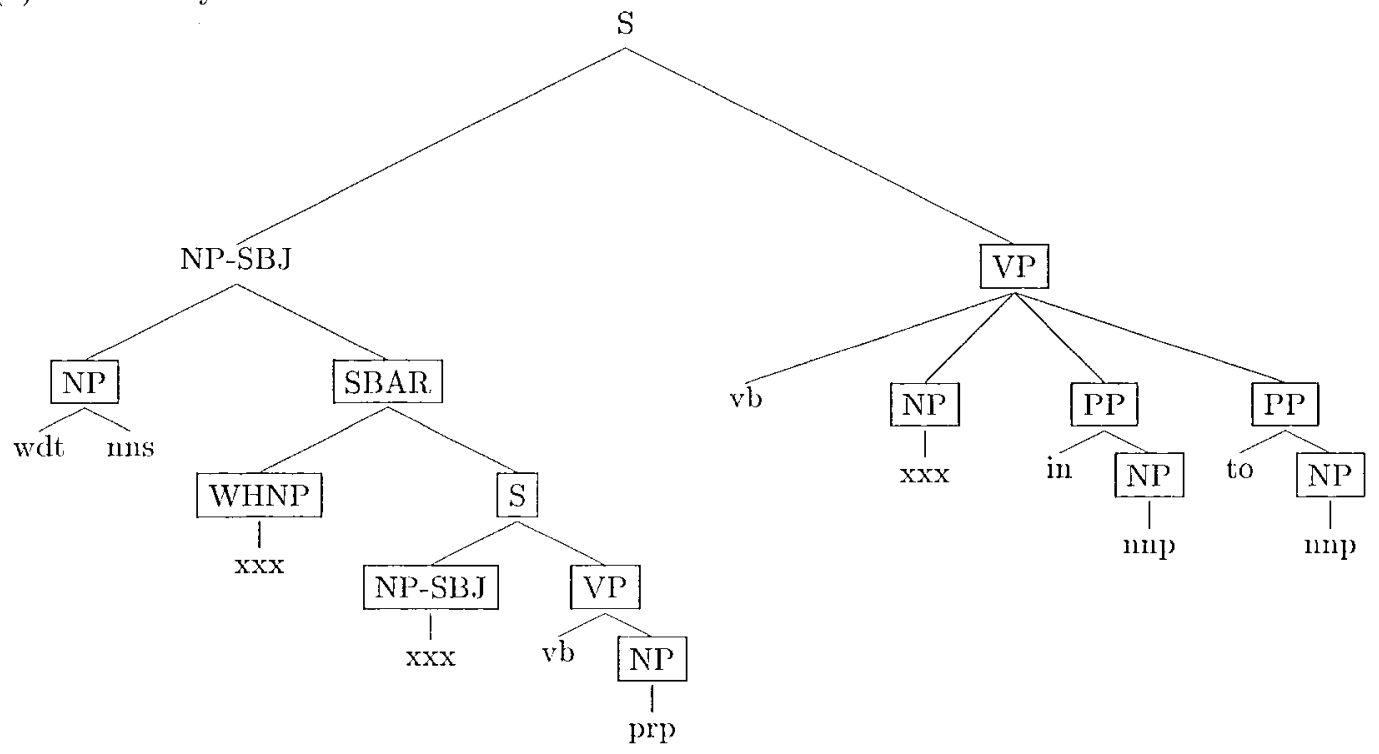

Figure 3: PMPG Error Analysis

Based Learner, version 2.0, reference manual. Technical Report ILK-9901, ILK, Tilburg University.

Daelemans, Walter. 1999. Memory-based language processing. Journal for Experimental and Theorctical Artificial Intelligence, 11:3:287-467.

De Pauw, Guy. 2000. Probabilistische Parsers - Contextgevoeligheid on Pattern-Matching. Antwerpen, Belgium: Antwerp Papers in Linguistics.

Goodman, Joshua. 1996. Efficient algorithms for parsing the dop model. In Proceedings of the Conference on Empirical Methods in Natural Language Processing. pages 143-152.

Marcus, M., B. Santorini, and M.A. Marcinkiewic\%. 1993. Building a large annotated corpus of english: The Penn Trecbank. Computational Linguistics, 19(2):313-330.
Scha, R. 1990. Taaltheoric en taaltechnologic: competence en performance. In Q. A. M. de Kort and G. L. J. Lcerdam, editors, Computertocpassingen in de Necrlandistick, LVVN-jaarbock. Landelijke Vereniging van Necrlandici.

van Halteren, H., J. Zavrel, and W. Daclemans. 1998. Improving data-driven wordclass tagging by system combination. In Proceedings of the 36 th Annual Mecting of the Association for Computational Linguistics, Montr'eal, Qucbec, Canada, pages 491-497, Montreal, Canada, August 10-14. 